\title{
CAN ONE CLASSIFY FINITE POSTNIKOV PIECES?
}

\author{
JESPER M. MØLLER AND JÉRÔME SCHERER
}

\begin{abstract}
We compare the classical approach of constructing finite Postnikov systems by $k$ invariants and the global approach of Dwyer, Kan, and Smith. We concentrate on the case of 3-stage Postnikov pieces and provide examples where a classification is feasible. In general though the computational difficulty of the global approach is equivalent to that of the classical one.
\end{abstract}

... all mathematics leads, doesn't it, sooner or later, to some kind of human suffering.

"Against the Day", Thomas Pynchon

\section{INTRODUCTION}

Let $X$ be a finite Postnikov piece, i.e. a space with finitely many non-trivial homotopy groups. Let us also assume for simplicity that $X$ is simply connected. The classical theory of $k$-invariants tells us that one can construct $X$ from Eilenberg-Mac Lane spaces and a finite number of cohomology classes, the $k$-invariants, but of course it might be difficult to compute them explicitly. This computational difficulty is probably best illustrated by how embarrassingly little one knows about the cohomology of Postnikov pieces which are not $H$-spaces, see [23] for one of the few examples where "something" has been computed.

In [9], Dwyer, Kan, and Smith propose a global approach. They provide in particular a model for the classifying space of finite towers $X_{n} \rightarrow X_{n-1} \rightarrow \cdots \rightarrow X_{1}$ in which each fiber is a given Eilenberg-Mac Lane space. We specialize to the case of 3-stage Postnikov pieces, and even further to fibrations of the form

$$
K(C, r) \times K(B, n) \rightarrow X \rightarrow K(A, m)
$$

with $1<m<n<r$. There exists a quite substantial literature about this situation, let us mention especially Booth's work, [3] and [4], and Pavešić, [21], [22]. We explain first how the Dwyer-Kan-Smith model provides a classifying space for such fibrations and show in Corollary 5.4 that it coincides with Booth's model from [3].

In the last section we compare then these two approaches, the classical one based on $k$-invariants and the global one, and show that they are basically equivalent. From the global point of view what we must compute is a set of homotopy classes of lifts in a fibration where the fiber is a product of Eilenberg-Mac Lane spaces. It is quite remarkable how difficult it is to compute this, compared to

2000 Mathematics Subject Classification. Primary 55S45; Secondary 55R15, 55R70, 55P20, 22F50.

The second author is supported by FEDER/MEC grant MTM2007-61545 and by the MPI, Bonn. 
the elementary case when the fiber is a single Eilenberg-Mac Lane space, a situation studied by the first author in [18, and completely understood.

Consequently, a classification of three-stage Postnikov pieces will be hopeless in general since it would necessitate the knowledge of the cohomology of an arbitrary two-stage Postnikov piece. However, classifications can be obtained in specific situations, and we provide such examples along the way. The fact that we could not find explicit computations in the literature motivated us to write this note (one exception which we learned from Fernando Muro is Baues' 2, Section 10.5]). Let us conclude with the comment that this project grew up from a desire to understand the real scope of the global classifying space model. Even though our conclusion might seem rather pessimistic from a computational point of view, we hope that the elegance of the global approach is still visible. Moreover, our arguments do not exclude the possibility that non-spectral sequence methods, applied to specific classes of Postnikov pieces, may sometimes work better in the global context than they do in the classical approach.

Acknowledgements. This work was achieved while the second author was visiting the MaxPlanck-Institut für Mathematik in Bonn. We would like to thank Christian Ausoni and the referee for helpful comments.

\section{Monoids of SELF-EQuivalences}

Let $X$ be a simply connected space. We consider some group-like topological monoids consisting of (homotopy classes of) self homotopy equivalences of $X$ :

$\operatorname{aut}(X)$ : the topological monoid of self-homotopy equivalences of $X$,

$\operatorname{aut}_{*}(X)$ : the topological monoid of pointed self-homotopy equivalences of $X$, $\operatorname{Aut}(X)$ : the discrete group of components of aut $(X)$.

In all cases the topological monoid structure is defined by composition of maps. If $X$ happens to be an $H$-space, such as a product of Eilenberg-MacLane spaces, then aut $(X)$ also inherits an $H$-space structure from $X$. These two structures are in general not the same.

Proposition 1.1. ([1], [26, [16, Chapter IV]) There is a bijection of sets of homotopy classes of unpointed maps $Y \rightarrow B$ aut $(X)$ and fiberwise homotopy types of fibrations of the form $X \rightarrow E \rightarrow Y$.

If $t: Y \rightarrow B$ aut $(X)$ classifies such a fibration, one often write $E=Y \times_{t} X$ for the total space and calls it a twisted product. Much attention has been received by the set of components $\operatorname{Aut}(X)$, but not so much by the space $\operatorname{aut}(X)$ itself. A nice exception is Farjoun and Zabrodsky's 8$]$.

\section{Reminder on 2-Stage Postnikov systems}

In any introductory book on homotopy theory, such as [30, Chapter IX], one can read that a simply connected space $E$ with only two non-trivial homotopy groups (say $\pi_{m} E \cong A$ and $\pi_{n} E \cong B$ 
for $n>m)$ is classified by a $k$-invariant $k: K(A, m) \rightarrow K(B, n+1)$. This means that $E$ has the homotopy type of the homotopy fiber of $k$. How does this relate to the approach described in the previous section?

We wish to understand the monoid aut $(K(B, n))$ and its classifying space. From Proposition 1.1 we infer that two-stage Postnikov pieces $E$ with $\pi_{m} E \cong A$ and $\pi_{n} E \cong B$ are in bijection with $[K(A, m), B$ aut $(K(B, n))]$.

As a space aut $(K(B, n))$ is a product $\operatorname{Aut}(B) \times K(B, n)$; this splitting is compatible with the $H$-space structure coming from that of the Eilenberg-Mac Lane space, but not with the one we are looking at, coming from composition. In fact $\operatorname{aut}_{*}(K(B, n))$ is weakly equivalent to the discrete monoid $\operatorname{Aut}(K(B, n)) \cong \operatorname{Aut}(B)$. The weak equivalence is given by functoriality of the $K(-, n)$ construction. Let us write $\varphi(\alpha)$ for the pointed self-equivalence associated to the group automorphism $\alpha$. The map $\varphi$ splits the monoid map $\pi_{n}$ : aut $(K(B, n)) \rightarrow \operatorname{Aut}(B)$. The fiber of $\pi_{n}$ over the identity is $\operatorname{aut}_{1}(K(B, n)) \simeq K(B, n)$, on which $\operatorname{Aut}(B)$ acts via $\varphi$ by conjugation. Thus we obtain a description of the classifying space, see [20].

Lemma 2.1. The split exact sequence $K(B, n) \rightarrow \operatorname{aut}(K(B, n)) \rightarrow \operatorname{Aut}(B)$ of topological monoids induces a split fibration

$$
K(B, n+1) \longrightarrow B \operatorname{Baut}(K(B, n)) \rightleftarrows B \operatorname{Aut}(B)
$$

and thus Baut $(K(B, n))$ is the classifying space for $(n+1)$-dimensional cohomology with local coefficients in $B$.

Proof. The section given by functoriality of the construction of Eilenberg-Mac Lane spaces is a map of monoids.

We recover now the classification of fibrations with fibers $K(B, n)$ as obtained by Steenrod in [27, Section 23] and Dold [7, Satz 12.15]. They are classified by a single $k$-invariant modulo the (non-trivial) action of $\operatorname{Aut}(B)$.

Theorem 2.2. The set of homotopy equivalent fibrations over a simply connected space $Y$ with fibers $K(B, n)$ is in bijective correspondance with $[Y, K(B, n+1)] / \operatorname{Aut}(B) \cong H^{m+1}(Y ; B) / \operatorname{Aut}(B)$.

Proof. If we apply the functor $[Y,-]$ to the fibration from Lemma 2.1, we obtain an exact sequence $[Y, \operatorname{Aut}(B)]=\operatorname{Aut}(B) \rightarrow[Y, K(B, n+1)]=H^{n+1}(Y ; B) \rightarrow[Y, B$ aut $(K(B, n))] \rightarrow *$ of sets and group actions.

Corollary 2.3. Let $n>m>1$ and $A, B$ be abelian groups. The set of $K(B, n)$-fiber homotopy types over $K(A, m)$ is in bijection with $H^{n+1}(K(A, m) ; B) / \operatorname{Aut}(B)$.

Let us look at a basic example, which will serve as starting point for examples of 3-stage Postnikov pieces. 
Example 2.4. For $m=2$ and $n=3$, let us choose $A=B=\mathbb{Z} / 2$ so $\operatorname{Aut}(\mathbb{Z} / 2)=1$. Since $H^{4}(K(\mathbb{Z} / 2,2) ; \mathbb{Z} / 2) \cong \mathbb{Z} / 2$, there are two homotopy spaces with the prescribed homotopy groups, namely the product $K(\mathbb{Z} / 2,2) \times K(\mathbb{Z} / 2,3)$ and $E_{2}$ the homotopy fiber of $S q^{2}: K(\mathbb{Z} / 2,2) \rightarrow$ $K(\mathbb{Z} / 2,4)$, the space studied in [17] by Milgram (and many others).

\section{The Classical approach to 3-stage Postnikov systems}

In principle, the above theorem (and its corollary) can be used inductively to classify $n$-stage Postnikov pieces. For a space $E$ with only three non-trivial homotopy groups $\pi_{m} E \cong A, \pi_{n} E \cong B$, and $\pi_{r} E \cong C$ for $r>n>m>1$ we could first construct $E[n]$, the $n$-th Postnikov section, which is given by an element in $H^{m+1}(K(A, m) ; B) / \operatorname{Aut}(B)$ by Corollary 2.3. To reconstruct $E$ we will then need to know the cohomology of $E[n]$, since the next $k$-invariant lives in $H^{r+1}(E[n] ; C) / \operatorname{Aut}(C)$. Our aim is to study fiber homotopy types where the fiber is a product of two Eilenberg-Mac Lane spaces.

Amazingly enough, we could not find a single example of classification of 3-stage Postnikov systems in the literature, except [2, Section 10.5] where Baues deals with $(n-1)$-connected $(n+2)$ types with $n \geq 4$. Let us treat thoroughly one example, where we do the computations "by hand". Its interest also lies in the kind of computation one has to perform in order to do the classification.

Example 3.1. Let us analyze fiber homotopy types of the form

$$
K(\mathbb{Z} / 2,5) \times K(\mathbb{Z} / 2,3) \rightarrow E \rightarrow K(\mathbb{Z} / 2,2)
$$

Thus $E$ has three non-trivial homotopy groups, all of them isomorphic to $\mathbb{Z} / 2$. There are two $k$ invariants. The first one is a cohomology class $k_{1} \in H^{4}\left(K(\mathbb{Z} / 2,2) ; \mathbb{F}_{2}\right) \cong \mathbb{F}_{2}\left\langle S q^{2} \iota_{2}\right\rangle$. Then the third Postnikov section $E[3]$ is the homotopy fiber of $k_{1}$ and the second $k$-invariant $k_{2} \in H^{6}\left(E[3] ; \mathbb{F}_{2}\right)$ is a class which restricts to zero in $H^{6}\left(K(\mathbb{Z} / 2,3) ; \mathbb{F}_{2}\right)$ since we want the 3 -connected cover $E\langle 3\rangle$ to split as a product $K(\mathbb{Z} / 2,5) \times K(\mathbb{Z} / 2,3)$.

When $k_{1}=0, k_{2}$ is a class in $H^{6}\left(K(\mathbb{Z} / 2,2) \times K(\mathbb{Z} / 2,3) ; \mathbb{F}_{2}\right)$ restricting to zero over $K(\mathbb{Z} / 2,3)$. By the Künneth formula we see that $k_{2}$ lies in

$$
H^{6}(K(\mathbb{Z} / 2,2)) \oplus H^{3}(K(\mathbb{Z} / 2,2)) \otimes H^{3}(K(\mathbb{Z} / 2,3)) \oplus H^{2}(K(\mathbb{Z} / 2,2)) \otimes H^{4}(K(\mathbb{Z} / 2,3)) .
$$

There are thus 16 possible $k$-invariants, i.e. 16 different fiber homotopy types of spaces $E$ over $K(\mathbb{Z} / 2,2) \times K(\mathbb{Z} / 2,3)$ with fiber $K(\mathbb{Z} / 2,5)$ such that $E\langle 3\rangle \simeq K(\mathbb{Z} / 2,5) \times K(\mathbb{Z} / 2,3)$. This is not quite what we want. The group of components $\mathbb{Z} / 2$ of $\operatorname{aut}(K(\mathbb{Z} / 2,2) \times K(\mathbb{Z} / 2,3))$ acts on the 16 $k$-invariants by composition. It is easy to compute explicitly this action of $\mathbb{Z} / 2$ : It acts trivially on 8 classes and identifies 4 pairs, so that we are left with 12 fiber homotopy types over $K(\mathbb{Z} / 2,2)$.

When $k_{1}=S q^{2} \iota_{2}$, let us denote by $E_{2}$ the homotopy fiber. The mod 2 cohomology of this space has been computed by Milgram, 17, or Kristensen and Pedersen, [12. It is an elementary Serre spectral sequence (for the fibration $K(\mathbb{Z} / 2,3) \rightarrow E_{2} \rightarrow K(\mathbb{Z} / 2,2)$ ) argument to compute it in low 
degrees. We denote by $\iota_{n}$ the non-zero class in $H^{n}\left(K(\mathbb{Z} / 2, n) ; \mathbb{F}_{2}\right)$. In total degree 6 , the only elements that survive are on the vertical axis $-H^{6}\left(K(\mathbb{Z} / 2,3) ; \mathbb{F}_{2}\right)-$ and the $\iota_{2} \otimes S q^{1} \iota_{3}$ in bidegree $(2,4)$.

As the second $k$-invariant is a class in $H^{6}\left(E_{2}\right)$ restricting to zero over $K(\mathbb{Z} / 2,3)$, it must be either zero or the class corresponding to $\iota_{2} \otimes S q^{1} \iota_{3}$. There are thus only 2 fiber homotopy types over $E_{2}$. Now, in principle, there could be an action of the group of self-equivalences of $E_{2}$ (isomorphic to $\mathbb{Z} / 2$ ) on these two $k$-invariants, but as it fixes zero, this action must be trivial. We have therefore also two fiber homotopy types over $K(\mathbb{Z} / 2,2)$ covering $S q^{2} \iota_{2}$.

The point of the example is that it illustrates well that one needs to know the cohomology in low degrees of certain 2-stage Postnikov systems (and then identify the action of a group of self-equivalences). This was easy here, but imagine the situation if one would wish to compute fiber homotopy type over $K(\mathbb{Z} / 2,2)$ with fiber $K(\mathbb{Z} / 2,3) \times K(\mathbb{Z} / 2,1000)$, or worse, to obtain a classification in cases where the first $k$-invariant is not primitive (say Schochet's [23] homotopy fiber of the map $K(\mathbb{Z} / 2 \oplus \mathbb{Z} / 2,2) \rightarrow K(\mathbb{Z} / 2,4)$, represented in cohomology by the product of the fundamental classes)!

\section{SpaCes of LifTS}

In this section we recall briefly the description and notation of certain spaces of lifts from the work of the first author in [18. It deals with the case when the fiber is a single Eilenberg-Mac Lane space. We then set up a spectral sequence to treat the case of a Postnikov piece. Even in the case when the fiber is a product of Eilenberg-Mac Lane spaces the description becomes quickly complex. We start with some generalities about spaces of lifts. Let us fix a fibration $p: Y \rightarrow Z$ and a map $u: X \rightarrow Y$.

Definition 4.1. The fiber containing $u \in \operatorname{map}(X, Y)$ of the induced fibration $\underline{p}: \operatorname{map}(X, Y) \rightarrow$ $\operatorname{map}(X, Z)$ is the space of lifts $\operatorname{map}(X, \emptyset ; Y, Z)_{u}=\{v \in \operatorname{map}(X, Y) \mid p v=p u\}$ of all maps lying over $p u$.

Let $p_{*}:[X, Y] \rightarrow[X, Z]$ be the induced map of sets of homotopy classes of maps.

Lemma 4.2. One has $[X, Y] \cong \coprod_{p_{*} u \in p_{*}[X, Y]} \pi_{0}\left(\operatorname{map}(X, \emptyset ; Y, Z)_{u}\right) / \pi_{1}(\operatorname{map}(X, Z), p u)$.

Proof. There are fibrations $\operatorname{map}(X, Y, Z)_{u} \rightarrow \operatorname{map}(X, Y)_{p_{*}^{-1}\left(p_{*} u\right)} \rightarrow \operatorname{map}(X, Z)_{p_{*} u}$ where $p_{*} u$ runs through the set $p_{*}[X, Y] \subset[X, Z]$.

In the associated action

$$
\pi_{1}(\operatorname{map}(X, Z), p u) \times \pi_{0}\left(\operatorname{map}(X, \emptyset ; Y, Z)_{u}\right) \longrightarrow \pi_{0}\left(\operatorname{map}(X, \emptyset ; Y, Z)_{u}\right)
$$


the effect of an element $[h] \in \pi_{1}(\operatorname{map}(X, Z), p u)$ of the fundamental group of the base space on the fibre $\operatorname{map}(X, \emptyset ; Y, Z)_{u}$ is given by $\bar{h}: \operatorname{map}(X, \emptyset ; Y, Z)_{u} \rightarrow \operatorname{map}(X, \emptyset ; Y, Z)_{u}$ where $\bar{h}$ is a lift

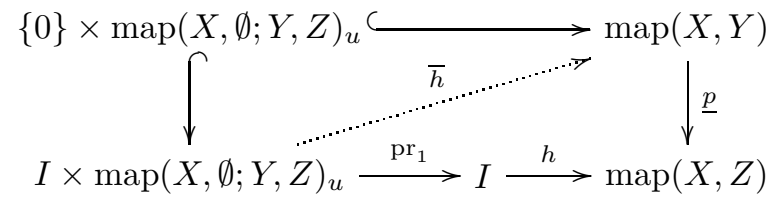

of the homotopy $h \circ \mathrm{pr}_{1}$. Equivalently, $\bar{h}$ is a solution to the adjoint homotopy lifting problem $I \times \operatorname{map}(X, \emptyset ; Y, Z)_{u} \times X \rightarrow Y$. Thus $\bar{h}$ is a homotopy from the evaluation map $\bar{h}(0, v, x)=v(x)$ such that $p \bar{h}(t, v, x)=h(t, x)$ is the given self-homotopy of $p u: X \rightarrow Z$. The end value of $\bar{h}$ takes $\operatorname{map}(X, \emptyset ; Y, Z)_{u}$ to itself.

Assume now that the fibre of the fibration $p: Y \rightarrow Z$ is the Eilenberg-Mac Lane space $K(A, n)$. The primary difference between the two lifts

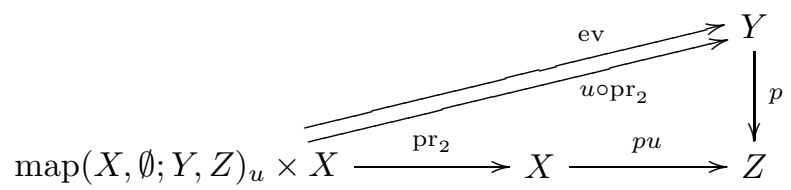

is an element of $\delta^{n}\left(\mathrm{ev}, u \circ \mathrm{pr}_{2}\right)$ in the group $H^{n}\left(\operatorname{map}(X, \emptyset ; Y, Z)_{u} \times X ; A\right)$. Let $\delta_{i}$ be the components in $\prod H^{i}\left(\operatorname{map}(X, \emptyset ; Y, Z)_{u} ; H^{n-i}(X ; A)\right)$ of $\delta^{n}\left(\mathrm{ev}, u \circ \mathrm{pr}_{2}\right)$ under the isomorphism

$$
H^{n}\left(\operatorname{map}(X, \emptyset ; Y, Z)_{u} \times X ; A\right) \cong \prod_{0 \leq i \leq n} H^{i}\left(\operatorname{map}(X, \emptyset ; Y, Z)_{u} ; H^{n-i}(X ; A)\right)
$$

for the cohomology of a product. We can now state the generalization of Thom's result, 29], obtained by the first author.

Theorem 4.3. (Møller, [18]) The map $\prod \delta_{i}: \operatorname{map}(X, \emptyset ; Y, Z)_{u} \rightarrow \prod_{0 \leq i \leq n} K\left(H^{n-i}(X ; A), i\right)$ is a homotopy equivalence.

In particular, $\pi_{0}\left(\operatorname{map}(X, \emptyset ; Y, Z)_{u}\right) \cong H^{n}(X ; A)$ and the action (11) takes the form of an action

$$
\pi_{1}(\operatorname{map}(X, Z), p u) \times H^{n}(X ; A) \rightarrow H^{n}(X ; A)
$$

of the group $\pi_{1}(\operatorname{map}(X, Z), p u)$ on the set $H^{n}(X ; A)$. How can we describe this action?

Lemma 4.4. Let ev $\in H^{n}(\operatorname{map}(X, K(A, n)) \times X ; A)$ be the evaluation map. Write $\mathrm{ev}=\sum \mathrm{ev}_{i}$ as a sum of cohomology classes under the Künneth isomorphism

$$
H^{n}(\operatorname{map}(X, K(A, n)) \times X ; A) \cong \bigoplus_{i+j=n} H^{i}\left(\operatorname{map}(X, K(A, n)) ; H^{j}(X ; A)\right)
$$

Then

$$
\prod \mathrm{ev}_{i}: \operatorname{map}(X, K(A, n)) \rightarrow \prod_{i+j=n} K\left(H^{j}(X ; A), i\right)
$$


is a homotopy equivalence.

We are now ready for the promised spectral sequence computing the homotopy groups of the space of lifts in a fibration where the fiber has more than a single non-trivial homotopy group (there is an analogous spectral sequence when the source $X$ is a finite CW-complex). It is obtained by decomposing the fiber by its Postnikov sections. The case of a space of sections has been studied in great detail by Legrand, [14. It generalizes work of Shih, [24], on limited and non-abelian spectral sequences. The bigrading we have chosen here agrees with that in [19, Theorem 5.3].

Corollary 4.5. Suppose that $F \rightarrow Y \rightarrow Z$ is a split fibration where the fibre $F$ is a finite Postnikov piece, connected and simple. Let $u: X \rightarrow Z$ be a map. Then there is third octant homology spectral sequence $(i+j \geq 0$ and $i \leq 0)$

$$
E_{i j}^{2}=H^{-i}\left(X ; \pi_{j}(F)\right) \Longrightarrow \pi_{i+j}\left(\operatorname{map}(X, \emptyset ; Y, Z)_{u}\right)
$$

converging to the homotopy groups of the space of lifts.

In principle, the cohomology groups appearing in the spectral sequence are to be understood with local coefficients defined by the choice of a lift. The space of lifts here is not empty since we assume for simplicity that the fibration has a section. The case when the fiber has two non-trivial homotopy groups is already interesting.

Example 4.6. Suppose that the fibre $F=K(A, m) \times K(B, n)$ with $m<n$. In that case the spectral sequence is concentrated on two lines and yields a long exact sequence. It can be identified with the homotopy long exact sequence of the fibration

$$
\operatorname{map}(Z, \emptyset ; Y, \bar{Y}[m])_{u} \longrightarrow \operatorname{map}(Z, \emptyset ; Y, Z)_{u} \longrightarrow \operatorname{map}(Z, \emptyset ; \bar{Y}[m], Z)_{u}
$$

where $\bar{Y}[m]$ denotes the fiberwise Postnikov section, i.e. the map $Y \rightarrow Z$ factors through $\bar{Y}[m]$ and the homotopy fiber of $\bar{Y}[m] \rightarrow Z$ is $F[m]=K(A, m)$. We deduce from Theorem 4.3 that $\operatorname{map}(Z, \emptyset ; Y, \bar{Y}[m])_{u} \simeq \prod K\left(H^{n-i}(Z ; B), i\right)$ and $\operatorname{map}(Z, \emptyset ; \bar{Y}[m], Z)_{u} \simeq \prod K\left(H^{m-i}(Z ; A), i\right)$. Hence the long exact sequence terminates in particular with

$$
H^{m-1}(X ; A) \rightarrow H^{n}(X ; B) \rightarrow \pi_{0} \operatorname{map}(Z, \emptyset ; Y, Z)_{u} \rightarrow H^{m}(X ; A)
$$

Note that even though the fibre is a product, the $k$-invariant $Y[m] \rightarrow K(B, n+1)$ may not be trivial (it only restricts to 0 on the fibre) and therefore the $k$-invariant of the above fibration may not be trivial either so that the sequence does not split!

This indicates that, as soon as there are more than one non-trivial homotopy group in the fiber, it will be difficult even to compute the number of homotopy classes of lifts, in contrast with Theorem 4.3 . 


\section{The Dwyer-Kan-Smith MOdel}

Let us now look at the "global" point of view on Postnikov pieces. Instead of adding iteratively one Eilenberg-Mac Lane space at a time, one can also try to understand how to add all homotopy groups at once. This is the approach followed by Dwyer, Kan, and Smith in [9]. In this section we will see how it specializes to the case of 3-stage Postnikov pieces and which modifications we need to obtain explicit classification results.

Let $G$ be a space and consider the functor $\Phi$ which sends an object of Spaces $\downarrow B$ aut $(G)$, i.e. a map $t: X \rightarrow B$ aut $(G)$, to the twisted product $X \times_{t} G$, see Section 11 Dwyer, Kan, and Smith describe a right adjoint $\Psi$ in [9, Section 4]. They find first a model for aut $(G)$ which is a (simplicial) group and thus acts on the left on $\operatorname{map}(G, Z)$ for any space $Z$. This induces a map $r: B$ aut $(G) \rightarrow B$ aut $(\operatorname{map}(G, Z))$. The functor $\Psi$ then sends $Z$ to the projection map from the twisted product, $B$ aut $(G) \times_{r} \operatorname{map}(G, Z) \rightarrow B$ aut $(G)$. This allows us to immediately construct a classifying space for towers, in our case they will be of length 2 .

Theorem 5.1. (Dwyer, Kan, Smith, [9]) The classifying space for towers of the form $Z \stackrel{q}{\rightarrow} Y \stackrel{p}{\rightarrow} X$, where the homotopy fiber of $p$ is $G$ and that of $q$ is $H$, is $B \operatorname{aut}(G) \times_{r} \operatorname{map}(G, B$ aut $(H))$.

Fix now a fibration $H \rightarrow F \rightarrow G$ where we think about the spaces $H$ and $G$ as simpler, in particular the spaces aut $(H)$ and aut $(G)$ should be accessible. Such a fibration is classified by a map $s: G \rightarrow B$ aut $(H)$ and so $F$ is the twisted product $G \times{ }_{s} H$. To construct $B$ aut $(F)$, one simply needs to refine a little the analysis done by Dwyer, Kan, and Smith. Let us denote by $\operatorname{map}(G, B \text { aut }(H))_{[s]}$ the components of the mapping space corresponding to the orbit of the map $s$ defined above under the action of $\operatorname{Aut}(G)$.

Lemma 5.2. Let $H \rightarrow F \rightarrow G$ be any fibration, classified by a map $s: G \rightarrow B$ aut $(H)$. The space $B$ aut $(G) \times_{r} \operatorname{map}(G, B \text { aut }(H))_{[s]}$ classifies towers $Z \stackrel{\beta}{\rightarrow} Y \stackrel{\alpha}{\rightarrow} X$ where the homotopy fiber of $\alpha$ is $G$, that of $\beta$ is $H$, and that of the composite $\alpha \circ \beta$ is $F$.

Proof. Since $B$ aut $(G) \times_{t} \operatorname{map}(G, B \text { aut }(H))_{[s]}$ is a subspace of the classifying space for towers $Z \rightarrow Y \rightarrow X$ over $X$ with fibers $G$ and $H$, it classifies some of them. We claim that the fiber of the composite map $Z \rightarrow X$ is precisely $F$.

From the adjunction property a map $X \rightarrow B$ aut $(G) \times_{r} \operatorname{map}(G, B \text { aut }(H))_{[s]}$ corresponds to a map $t^{\prime}: X \times{ }_{t} G \rightarrow B$ aut $(H)$, which yields a space $E=X \times_{t} G \times{ }_{t^{\prime}} H$. The fiber we must identify is thus the homotopy pull-back of the diagram $E \rightarrow X \times{ }_{t} G \leftarrow G$. In other words it is the twisted product corresponding to the composite map $G \rightarrow X \times_{t} G \rightarrow B$ aut $(H)$, which is homotopic to $s$. This means that the homotopy fiber is $F$.

To find an description of $B$ aut $(F)$ in terms of $G$ and $H$ is a more difficult task, because in general not all fibrations with fiber $F$ come from a tower as above. However there are situations 
where this is so. Let us consider a homotopy localization functor $L$, like Postnikov sections, Quillen plus-construction, or localization at a set of primes, see [10]. What matters for us is that there are natural maps $\eta: X \rightarrow L X$ for all spaces $X$, and that $L$ sends weak equivalences to weak equivalences.

Theorem 5.3. Let $L$ be a homotopy localization functor and consider a fibration $\bar{L} F \rightarrow F \stackrel{\eta}{\rightarrow} L F$, classified by a map $s: L F \rightarrow B$ aut $(\bar{L} F)$. Then $B \operatorname{aut}(F)$ is $B \operatorname{aut}(L F) \times_{r} \operatorname{map}(L F, B \text { aut }(\bar{L} F))_{[s]}$.

Proof. Let $F \rightarrow Z \rightarrow X$ be any fibration over $X$. It is possible to construct a fiberwise version of $L$, i.e. obtain a new fibration $L F \rightarrow Y \rightarrow X$ such that the diagram

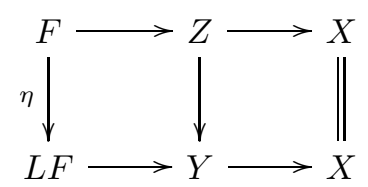

commutes, [10, Theorem F.3]. So any fibration comes from a tower $Z \rightarrow Y \rightarrow X$. In particular this construction can be applied to the universal fibration $F \rightarrow B$ aut $(F) \rightarrow B$ aut $(F)$ and this yields a map $B$ aut $(F) \rightarrow B$ aut $(L F) \times_{r} \operatorname{map}(L F, B$ aut $(\bar{L} F))$, which factors through the component $B$ aut $(L F) \times_{r} \operatorname{map}(L F, B \text { aut }(\bar{L} F))_{[s]}$ by Lemma 5.2. There is a forgetful map going the other way, and both composites are homotopic to the identity by uniqueness of the classifying space.

We are mainly interested in 3-stage Postnikov systems in this note. Consider thus a 3 -stage Postnikov piece $E$ as being the total space of a fibration of the form $F \rightarrow E \rightarrow K(A, m)$. The fiber $F$ is a space with only two non-trivial homotopy groups and the fibration is classified by a map $K(A, m) \rightarrow B$ aut $(F)$, see Theorem 1.1. We understand now the monoid of self-equivalences of a space with two non-trivial homotopy groups.

Corollary 5.4. Let $F$ be a simply connected 2-stage Postnikov piece, with $\pi_{m} F \cong A, \pi_{n} F \cong B$, $k$-invariant $k$, and $n>m$. Then $B$ aut $(F)$ is $B$ aut $(K(A, m)) \times{ }_{r} \operatorname{map}(K(A, m), B \operatorname{aut}(K(B, n)))_{[k]}$. Proof. The $m$-th Postnikov section $F \rightarrow F[m]$ is a homotopy localization functor.

Let us specialize even further, and assume that the $k$-invariant is trivial, that is, we are looking at a fiber which is a product of two Eilenberg-Mac Lane spaces. Such a model has been independently constructed by Booth in $[\underline{3}$.

Corollary 5.5. Let $A$ and $B$ be two abelian groups and $n>m$. Then $B$ aut $(K(A, m) \times K(B, n)) \simeq$ $B$ aut $(K(A, m)) \times_{r} \operatorname{map}(K(A, m), B \text { aut }(K(B, n)))_{c}$, where $c$ is the constant map. The projection $B$ aut $(K(A, m) \times K(B, n)) \rightarrow B$ aut $(K(A, m))$ has a section.

Proof. The orbit of the constant map is reduced to the constant map.

The computation of the set of components of aut $(K(A, m) \times K(B, n))$ is straightforward, compare with Shih's [25], or the matrix presentation used in [4, Section 1]. 
Corollary 5.6. Let $A$ and $B$ be two abelian groups and $n>m>1$ be integers. Then the group $\operatorname{Aut}(K(A, m) \times K(B, n))$ is a split extension of $\operatorname{Aut}(A) \times \operatorname{Aut}(B)$ by $H^{n}(K(A, m) ; B)$.

\section{Comparing the Classical With the Global APProACH}

The classical approach to finite $n$-stage Postnikov pieces goes through the computation of the cohomology of a $(n-1)$-stage Postnikov piece. This is theoretically feasible via a Serre spectral sequence computation, but practically very hard because of the differentials. What about the global approach?

We consider the case of fiber homotopy types over $K(A, m)$ with fiber $K(B, n) \times K(C, r)$ with $1<m<n<r$ as before. In principle we only need to compute the set of homotopy classes $[K(A, m), B \operatorname{aut}((K(B, n) \times K(C, r))]$ and we have a model for this classifying space. The only sensible way we could think of to compute this is by using the split fibration

$$
\operatorname{map}(K(B, n), B \operatorname{aut}(K(C, r)))_{c} \rightarrow B \operatorname{aut}((K(B, n) \times K(C, r)) \rightarrow B \operatorname{aut}(K(B, n))
$$

obtained in Corollary 5.5. Thus for each first $k$-invariant $k_{1}: K(A, m) \rightarrow K(B, n+1)$ we must understand the set of components of the space of lifts into $B$ aut $((K(B, n) \times K(C, r))$.

Example 6.1. Let us again analyze fiber homotopy types of the form

$$
K(\mathbb{Z} / 2,5) \times K(\mathbb{Z} / 2,3) \rightarrow E \rightarrow K(\mathbb{Z} / 2,2)
$$

We will now do the computation globally. Let us write shortly $K_{n}$ for the space $K(\mathbb{Z} / 2, n)$. The classifying space is $K_{4} \times_{t} \operatorname{map}\left(K_{3}, K_{6}\right)_{c}$. Consider now the sectioned fibration

$$
K_{6} \times K_{3} \times K_{2} \times K_{1}=\operatorname{map}\left(K_{3}, K_{6}\right)_{c} \longrightarrow \operatorname{Baut}\left(K_{3} \times K_{5}\right) \underset{s}{\rightleftarrows} \operatorname{Baut}\left(K_{3}\right)=K_{4}
$$

so that $\left[K_{2}, \operatorname{Baut}\left(K_{3} \times K_{5}\right)\right]$ is the disjoint union of the components of map $\left(K_{2}, \operatorname{Baut}\left(K_{3} \times K_{5}\right)\right)$ which lie over 0 and those which lie over $S q^{2} \iota_{2}$ in $\operatorname{map}\left(K_{2}, K_{4}\right)$. By Lemma 4.2 these two sets can be computed as quotients of sets of components of spaces of lifts under the action of a fundamental group.

Let us do that. Over zero, there is no mystery, the space of lifts is $\operatorname{map}\left(K_{2}, K_{6} \times K_{3} \times K_{2} \times K_{1}\right)$ and the fundamental group in question is $\pi_{1} \operatorname{map}\left(K_{2}, K_{4}\right) \cong \mathbb{Z} / 2$. It is straightforward to see that the 16 components of the mapping space are grouped in 12 orbits. Over $S q^{2} \iota_{2}$, we are looking at the space of lifts as in the following diagram:

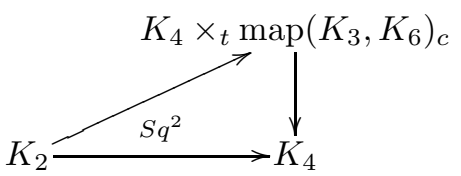

This is equivalent by the Dwyer-Kan-Smith adjunction [9, Section 4] to the subspace of maps $\operatorname{map}\left(E_{2}, K_{6}\right)$ which restrict trivially to $K_{3}$. From the 16 possible components we are left with two, 
compare with Example 4.6. The action of $\pi_{1} \operatorname{map}\left(K_{2}, K_{4}\right)_{S q^{2}} \cong \mathbb{Z} / 2$ is trivial and it seems we have redone here as well the same computation as in Example 3.1.

Let us carefully check whether we have really redone the same computations as in the classical approach.

Our typical study case is that of a space with three non-trivial homotopy groups $A, B$, and $C$, in degree respectively $m, n$, and $r$, with $1<m<n<r$. In the classical approach we use for each possible first $k$-invariant $k_{1}: K(A, m) \rightarrow K(B, n+1)$ the corresponding Serre spectral sequence $H^{p}\left(K(A, m) ; H^{q}(K(B, n) ; C)\right)$ of which we only need the $p+q=r+1$-diagonal to determine the possible values of the second $k$-invariant.

In the global approach we wish to compute, for each possible first $k$-invariant $k_{1}: K(A, m) \rightarrow$ $K(B, n+1)$, the set of components of the space of lifts indicated in the diagram

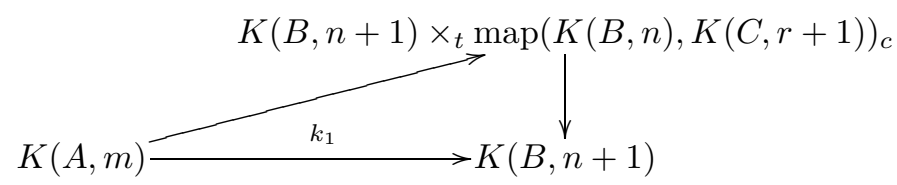

Since the mapping space $\operatorname{map}(K(B, n), K(C, r+1))_{c}$ is a product of Eilenberg-Mac Lane spaces $K\left(H^{n+1-j}(K(B, n) ; C), j\right)$ for $1 \leq j \leq n+1$, Corollary 4.5 yields a spectral sequence of the form $E_{2}^{i j}=H^{-i}\left(K(A, m) ; H^{r+1-j}(K(B, n) ; C)\right)$ with differential $d_{2}$ of bidegree $(-2,1)$.

Techniques due to Legrand, 14] and [13, allow to prove the following result.

Proposition 6.2. (Didierjean and Legrand, [6, Théorème 2.2]) Suppose that $F \rightarrow Y \rightarrow Z$ is a fibration where the fibre $F$ is a connected, finite Postnikov piece. The spectral sequences converging to the homotopy groups of the space of lifts $\operatorname{map}(X, \emptyset ; Y, Z)_{u}$ defined from the skeletal filtration of $X$, and the one defined by the Postnikov decomposition of $F$ are isomorphic.

Proof. The same argument as in [6 for spaces of sections applies for spaces of lifts. It relies on the techniques developed in [14. Alternatively one could identify the space of lifts as a space of sections (of the pull-backed fibration) and apply directly Didierjean and Legrand's result.

Remark 6.3. This kind of spectral sequence appeared maybe first in work of Federer, [11]. It also appears in Switzer, 28, in both forms, but he does not compare them however. When the target $Y$ is a spectrum rather than a space, the spectral sequences are the Atiyah-Hirzebruch one and the Postnikov one. Maunder proved they coincide, [15]. When $Y$ is a space, like here, cosimplicial technology allowed Bousfield to construct such spectral sequences yet in another way, 5 .

We now come back to our Postnikov pieces. The above proposition allows us to identify the spectral sequence coming from a Postnikov decomposition of map $(K(B, n), K(C, r+1))_{c}$ with the one coming from the skeletal filtration of $K(A, m)$. The last step is to identify this second spectral 
sequence. Let us first regrade the spectral sequence by setting $p=-i$ and $q=r+1-j$, so our $E_{2^{-}}$ term looks like $E_{2}^{p q}=H^{p}\left(K(A, m) ; H^{q}(K(B, n) ; C)\right)$ (and the differential $d_{2}$ has bidegree $(2,-1)$ ). This spectral sequence is concentrated in the first quadrant, in the horizontal band $0 \leq q \leq r+1$. It converges to $\pi_{p+q-r-1} \operatorname{map}\left(K(A, m), \emptyset ; K(B, n+1), K(B, n+1) \times_{t} \operatorname{map}(K(B, n), K(C, r+1))_{c}\right)_{k_{1}}$.

Theorem 6.4. Let $r>n>m>1$ be integers and $A, B, C$ be abelian groups. For any $k$ invariant $k_{1}: K(A, m) \rightarrow K(B, n+1)$, the part of the Postnikov spectral sequence concentrated in degrees $p+q \leq r+1$ computing the homotopy groups of the space of lifts into $K(B, n+1) \times_{t}$ $\operatorname{map}(K(B, n), K(C, r+1))_{c}$ over $k_{1}$ is isomorphic to the corresponding part of the cohomological Serre spectral sequence (with coefficients in $C$ ) for the fibration $K(B, n) \rightarrow K(A, m) \times_{k_{1}} K(B, n) \rightarrow$ $K(A, m)$.

Proof. The fiber map $(K(B, n), K(C, r+1))_{c}$ is a connected and finite Postnikov piece with abelian fundamental group, so that the Postnikov spectral sequence from Corollary 4.5 exists. From the previous proposition we know that it actually coincides with the spectral sequence defined by the skeletal filtration of $K(A, m)$.

Instead of looking at the $E_{2}$-term we will work with the $E_{1}$-term. We write $K(A, m)_{k} \subset K(A, m)$ for the $k$-th skeleton, and $p: K(A, m) \times_{k_{1}} K(B, n) \rightarrow K(A, m)$ for the natural projection. By the Dwyer-Kan-Smith adjunction lifts over $K(A, m)_{k}$ correspond to maps from the preimage under $p$ to $K(C, r+1)$. This is precisely the filtration in the Serre spectral sequence. All differentials in the triangle $p+q \leq r+1$ remain in the band $0 \leq q \leq r+1$, in which the $E_{2}$-term of the Postnikov sequence is abstractly isomorphic to the $E_{2}$-term of the Serre spectral sequence thanks to the regrading we have performed (for $q>r+1$ it is zero).

Remark 6.5. Let $r>n>m>1$ be integers and $A, B, C$ be abelian groups. We have seen two approaches to compute the number of fiber homotopy types $X$ over $K(A, m)$ with fiber $K(B, n) \times$ $K(C, r)$ such that $X[n]$ is classified by a given $k$-invariant $k_{1}: K(A, m) \rightarrow K(B, n+1)$. The one we have called the global one computes the set of components of a space of lifts via a Postnikov spectral sequence. Since the diagonal $p+q-r-1=0$ is contained (as the edge) in the triangle we have been able to analyze in Theorem 6.4 we see that this computation is exactly the same as the classical one, where one is looking for the second $k$-invariant.

\section{REFERENCES}

1. M. G. Barratt, V. K. A. M. Gugenheim, and J. C. Moore, On semisimplicial fibre-bundles, Amer. J. Math. 81 (1959), 639-657.

2. H.-J. Baues, Homotopy type and homology, Oxford Mathematical Monographs, The Clarendon Press Oxford University Press, New York, 1996, Oxford Science Publications.

3. P. I. Booth, Fibrations with product of Eilenberg-MacLane space fibres. I, Groups of homotopy self-equivalences and related topics (Gargnano, 1999), Contemp. Math., vol. 274, Amer. Math. Soc., Providence, RI, 2001, pp. 79104. 
4. _ An explicit classification of three-stage Postnikov towers, Homology, Homotopy Appl. 8 (2006), no. 2, 133-155 (electronic).

5. A. K. Bousfield, Homotopy spectral sequences and obstructions, Israel J. Math. 66 (1989), no. 1-3, 54-104.

6. A. Didierjean and A. Legrand, Suites spectrales de Serre en homotopie, Ann. Inst. Fourier (Grenoble) 34 (1984), no. $2,227-242$.

7. A. Dold, Halbexakte Homotopiefunktoren, Lecture Notes in Mathematics, vol. 12, Springer-Verlag, Berlin, 1966.

8. E. Dror and A. Zabrodsky, Unipotency and nilpotency in homotopy equivalences, Topology 18 (1979), no. 3, $187-197$.

9. W. G. Dwyer, D. M. Kan, and J. H. Smith, Towers of fibrations and homotopical wreath products, J. Pure Appl. Algebra 56 (1989), no. 1, 9-28.

10. E. Dror Farjoun, Cellular spaces, null spaces and homotopy localization, Lecture Notes in Mathematics, vol. 1622, Springer-Verlag, Berlin, 1996.

11. H. Federer, A study of function spaces by spectral sequences, Trans. Amer. Math. Soc. 82 (1956), 340-361.

12. L. Kristensen and E. K. Pedersen, The $\mathcal{A}$-module structure for the cohomology of two-stage spaces, Math. Scand. 30 (1972), 95-106.

13. A. Legrand, Sur les groupes d'homotopie des sections continues d'une fibration en groupes, C. R. Acad. Sci. Paris Sér. A-B 286 (1978), no. 20, A881-A883.

14. _ Homotopie des espaces de sections, Lecture Notes in Mathematics, vol. 941, Springer-Verlag, Berlin, 1982.

15. C. R. F. Maunder, The spectral sequence of an extraordinary cohomology theory, Proc. Cambridge Philos. Soc. 59 (1963), 567-574.

16. J. P. May, Simplicial objects in algebraic topology, Chicago Lectures in Mathematics, University of Chicago Press, Chicago, IL, 1992, Reprint of the 1967 original.

17. R. James Milgram, The structure over the Steenrod algebra of some 2-stage Postnikov systems, Quart. J. Math. Oxford Ser. (2) 20 (1969), 161-169.

18. J. M. Møller, Spaces of sections of Eilenberg-Mac Lane fibrations, Pacific J. Math. 130 (1987), no. 1, 171-186.

19. - On equivariant function spaces, Pacific J. Math. 142 (1990), no. 1, 103-119.

20. P. Olum, On mappings into spaces in which certain homotopy groups vanish, Ann. of Math. (2) 57 (1953), 561-574.

21. P. Pavešić, On the group $\operatorname{Aut}_{\#}\left(X_{1} \times \cdots \times X_{n}\right)$, Topology Appl. 153 (2005), no. 2-3, 485-492.

22. _ Reducibility of self-homotopy equivalences, Proc. Roy. Soc. Edinburgh Sect. A 137 (2007), no. 2, 389413.

23. C. Schochet, $A$ two-stage Postnikov system where $E_{2} \neq E_{\infty}$ in the Eilenberg-Moore spectral sequence, Trans. Amer. Math. Soc. 157 (1971), 113-118.

24. W. Shih, Homologie des espaces fibrés, Inst. Hautes Études Sci. Publ. Math. (1962), no. 13, 88.

25. - On the group $\mathcal{E}[X]$ of homotopy equivalence maps, Bull. Amer. Math. Soc. 70 (1964), 361-365.

26. J. Stasheff, A classification theorem for fibre spaces, Topology 2 (1963), 239-246.

27. N. E. Steenrod, Cohomology operations, and obstructions to extending continuous functions, Advances in Math. 8 (1972), 371-416.

28. R. M. Switzer, Counting elements in homotopy sets, Math. Z. 178 (1981), no. 4, 527-554.

29. R. Thom, L'homologie des espaces fonctionnels, Colloque de topologie algébrique, Louvain, 1956, Georges Thone, Liège, 1957, pp. 29-39.

30. G. W. Whitehead, Elements of homotopy theory, Graduate Texts in Mathematics, vol. 61, Springer-Verlag, New York, 1978. 
Jerôme Scherer

Departament de Matemàtiques,

Universitat Autònoma de Barcelona,

E-08193 Bellaterra, Spain

E-mail: jscherer@mat.uab.es
Jesper M. Møller

Matematisk Institut

Universitetsparken 5

DK-2100 København

E-mail:moller@math.ku.dk, 